Clinical Study

\title{
Novel Models for the Prediction of Left Atrial Appendage Thrombus in Patients with Chronic Nonvalvular Atrial Fibrillation
}

\author{
Do Van Chien (D), Pham Thai Giang, Pham Truong Son, Le Van Truong, \\ and Pham Nguyen Son \\ Department of Cardiology, Military Heart Institute, 108 Central Military Hospital, Hanoi, Vietnam \\ Correspondence should be addressed to Pham Nguyen Son; sonpna2@gmail.com
}

Received 3 April 2019; Accepted 8 July 2019; Published 25 August 2019

Academic Editor: Terrence D. Ruddy

Copyright ( 92019 Do Van Chien et al. This is an open access article distributed under the Creative Commons Attribution License, which permits unrestricted use, distribution, and reproduction in any medium, provided the original work is properly cited.

Predicting left atrial appendage thrombus (LAAT) in chronic nonvalvular atrial fibrillation remains challenging despite the fact that several predictive models have been proposed to date. In this study, we sought to develop new and simpler models for LAAT prediction in chronic nonvalvular atrial fibrillation. The study enrolled 144 patients with chronic nonvalvular atrial fibrillation who underwent transesophageal echocardiography for LAAT detection. We examined the association of LAAT incidence with the $\mathrm{CHA}_{2} \mathrm{DS}_{2}$-VASc score and echocardiographic parameters pertaining to the left atrium (LA), including diameter, volume index, strain, and strain rate measured on speckle tracking echocardiography. LAAT was found in $24.3 \%$ of patients (39/144). The following parameters had good diagnostic performance for LAAT: LA volume index $>57 \mathrm{~mL}$ (area under the curve (AUC), 0.72; sensitivity, $77.1 \%$; specificity, $64.2 \%$ ), LA positive strain $\leq 6.7 \%$ in the four-chamber view (AUC, 0.84 ; sensitivity, $77.1 \%$; specificity, 77.1\%), and LA negative strain rate $>-0.73 \mathrm{~s}^{-1}$ in the four-chamber view (AUC, 0.83; sensitivity, 85.7\%; specificity, 70.6\%). The $\mathrm{CHA}_{2} \mathrm{DS}_{2}$-VASc score alone had a low predictive value for LAAT in this population $\left(\chi^{2}=3.53\right)$, whereas the combination of $\mathrm{CHA}_{2} \mathrm{DS}_{2}$-VASc score with LA volume index had significant association and better predictive value $\left(\chi^{2}=12.03\right)$, and the combination of $\mathrm{CHA}_{2} \mathrm{DS}_{2}$-VASc score with LA volume index and LA positive strain or negative strain rate in the four-chamber view had the best predictive ability for LAAT ( $\chi^{2}: 33.47$ and 33.48 , respectively). We propose two novel and simple models for noninvasive LAAT prediction in patients with chronic nonvalvular atrial fibrillation. These models combine the $\mathrm{CHA}_{2} \mathrm{DS}_{2}$-VASc score with LA volume index and LA longitudinal strain parameters measured on speckle tracking echocardiography in the fourchamber view. We hope these simple models can help with decision-making in managing the antithrombotic treatment of such patients, whose risk of stroke cannot be determined solely based on the $\mathrm{CHA}_{2} \mathrm{DS}_{2}$-VASc score.

\section{Introduction}

Atrial fibrillation (AF) is the most prevalent arrhythmia in the general population, especially among the elderly [1]. In European countries, the prevalence of AF ranges from 1.9\% in Italy, Iceland, and England, to 2.3\% in Germany, and 2.9\% in Sweden. These numbers are expected to increase in the near future [2]. The prevalence of AF is lower in Asian than in Western countries, having been reported at approximately $1.6 \%$ in Japan [3] and $1.0 \%$ in Korea [4]. AF is associated with increased risk of ischemic stroke, systemic thromboembolism, and transient ischemic attack, which ranges from $1.5 \%$ in individuals aged $50-59$ years to $23.5 \%$ in individuals aged $80-89$ years [5]. Recent studies indicate that cardioembolic stroke accounts for $16 \%-30 \%$ of cases of ischemic stroke [6]. Compared to non-AF stroke, cardioembolic stroke due to AF is associated with higher risk of mortality and worse outcomes [7]. Therefore, it is of utmost importance to clarify the risk factors for AF stroke and adequately stratify patients according to stroke risk.

The left atrium (LA) is a specialized cardiac structure that generates and maintains chaotic electrical impulses in $\mathrm{AF}$, eventually losing contractility and causing atrial hemodynamic instability. The LA has a distinctive appendage shaped 
as a finger-like pouch extending from the main body of the LA and is considered the main source of clot formation in $\mathrm{AF}$ [8]. Therefore, it is important to adequately assess the LA appendage.

While cardiac computer tomography and magnetic resonance imaging are the most accurate methods for LA assessment, these methods are expensive and time-consuming and moreover require highly qualified expertise. Speckle tracking echocardiography (STE) is a new ultrasound-based modality designed for left ventricular assessment. However, recent studies have shown that STE can also be applied for measuring LA strain and strain rate [9-11]. Therefore, STE may be useful in screening AF patients for stroke risk.

Several STE-measurable parameters including LA diameter, LA volume index (LAVi), LA strain, and LA strain rate are known to be independently associated with stroke in AF patients [12-14]. However, the association of such parameters with thromboembolism in AF remains unclear. Therefore, in this study, we aimed to develop simple models for predicting the presence of LA appendage thrombus (LAAT) based on clinical factors and LA parameters measured on STE. Such models may help with decision-making in managing the antithrombotic treatment of patients with chronic nonvalvular AF.

\section{Materials and Methods}

2.1. Patients. From September 2013 to December 2017, we intentionally enrolled 144 anticoagulant-naïve patients aged 40-90 years and diagnosed with permanent nonvalvular AF defined according to the guidelines issued by the European Society of Cardiology in 2010 [15]. We excluded patients with moderate-to-severe rheumatic mitral stenosis with regurgitation, aortic stenosis with regurgitation, prosthetic valve, or previous surgical valvular repair. All patients underwent transesophageal echocardiography (TEE) to check for LAAT.

The study protocol was approved by our hospital's ethics committee, and all patients received extensive explanations regarding the risks associated with TEE. Informed consent was obtained from all patients before the procedure.

All patients were treated with AF rate control drugs to get their heart rates from 50 to 90 beats per minute and were carefully examined. The $\mathrm{CHA}_{2} \mathrm{DS}_{2}$-VASc score was calculated as follows: congestive heart failure, 1 point; hypertension, 1 point; age $\geq 75$ years, 2 points; diabetes type 2, 1 point; stroke or history of transient ischemic attack, 2 points; vascular disease (i.e., prior myocardial infarction, peripheral artery disease, or aortic plaque), 1 point; age 65-74 years, 1 point; female sex, 1 point.

\subsection{Transthoracic Echocardiography Evaluation.} Echocardiography was performed using a high-quality ultrasound machine (VIVID 7; GE Medical Systems, Milwaukee, WI, USA) equipped with a $1.7 / 3.4-\mathrm{MHz}$ tissue harmonic transducer. Electrocardiograms were recorded for all patients and synchronized with the echocardiographic images. Gain, depth, and focus position were adjusted to obtain a frame rate of 40-60 frames per second. Ultrasound beam width was set to obtain clear endocardial borders of the left ventricle and LA for offline processing and analysis. All images were acquired at the end of the expiratory period for three consecutive cardiac cycles and stored on a hard disk. All measurements were conducted with the patient in the left lateral decubitus position. STE data were analyzed using dedicated software. Speckle tracking and cardiac chamber measurements were conducted according to the guidelines issued by the American Society of Echocardiography and the European Association of Cardiovascular Imaging [16-19].

In the four- and two-chamber views, LA volume $(\mathrm{mL})$ was measured by tracing the endocardium and applying the area-length technique. Specifically, LA volume was calculated as $8 /(3 \pi)[(\mathrm{A} 1 \cdot \mathrm{A} 2) / L]$, where $\mathrm{A} 1$ is the LA area $\left(\mathrm{cm}^{2}\right)$ in the four-chamber view, A2 is the LA area $\left(\mathrm{cm}^{2}\right)$ in the twochamber view, and $L(\mathrm{~cm})$ is the shortest of the two long axes. Subsequently, the LAVi $\left(\mathrm{mL} / \mathrm{m}^{2}\right)$ was obtained as the LA volume $(\mathrm{mL})$ divided by the body surface area $\left(\mathrm{m}^{2}\right)$.

LA strain and strain rate were measured for the four- and two-chamber views of images obtained using two-dimensional STE and analyzed using EchoPAC (GE Medical Systems). EchoPAC was designed for echocardiographic assessment of the left ventricle [19]. However, recent studies demonstrated that EchoPAC can also be applied for LA speckle tracking analysis [20, 21]. After selecting an echo image at a specific cardiac cycle, the LA endocardium was traced manually from the mitral valve ring to the opposite end, ignoring the pulmonary vein. While epicardial borders could be detected automatically, some adjustments were sometimes necessary to cover the entire LA wall thickness. Before initiating image processing, the software requested the operator to confirm the selections and regions of interest. For each view, the software divided the LA into six segments (different color coding in Figure 1). The LA strain and strain rate were obtained separately for each segment and then averaged over all 12 segments.

2.3. TEE Evaluation. TEE was performed using the same ultrasound machine (VIVID 7; GE Medical Systems) equipped with the $3.5 / 7-\mathrm{MHz}$ multiplane probe. Patients fasted for at least 6 hours received local anesthesia using lignocaine spray and, if necessary, intravenous midazolam (3-5 mg) for sedation. With the patients in the left lateral decubitus position, the transducer was slowly advanced through the mouth guard into the esophagus. If any resistance was met, the direction of the probe was carefully shifted and advancement was resumed. If it was not possible to advance the probe, the procedure was stopped.

All cardiac chambers were surveyed carefully to search for thrombus in the LA and LA appendage. Thrombus was defined as a fixed or mobile echogenic mass clearly distinguishable from the wall of the LA or LA appendage. Spontaneous echo contrast was diagnosed as dynamic or "smoke-like" echo signal inside the LA that could not be eliminated by changing the gain settings [22]. 


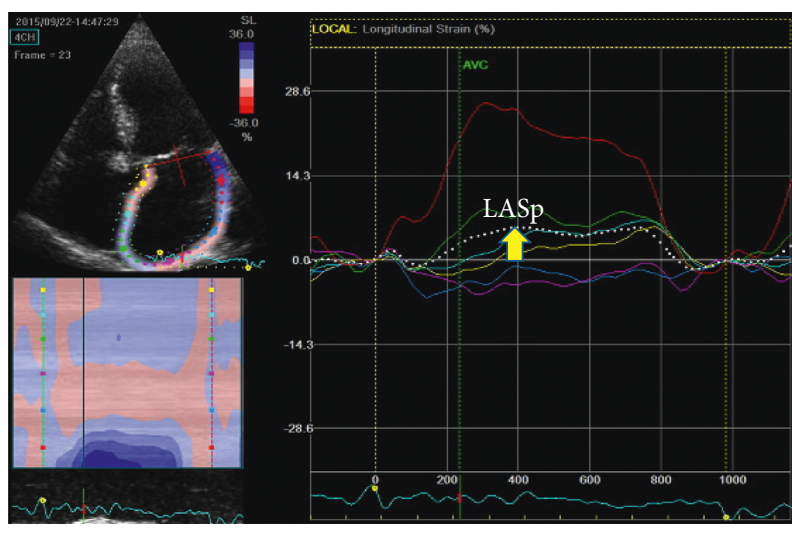

(a)

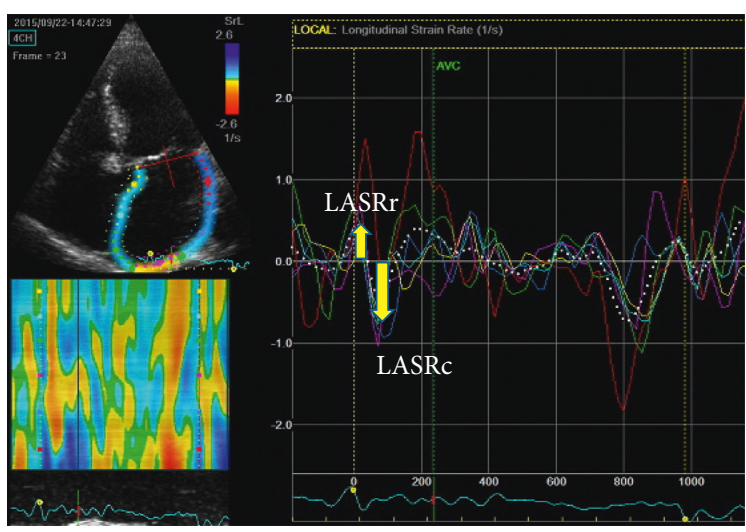

(b)

FIgURE 1: Measurement of left atrial (LA) strain and strain rate in the four-chamber view. (a) LA positive strain (LASp). (b) Evaluation of LA longitudinal strain rate was conducted separately for positive (LASRr) and negative (LASRc) values.

2.4. Reproducibility. Interobserver variablity and intraobserver variability for LA strain and strain rate were studied twice in a group of 10 randomly selected subjects by one operator and by two investigators who were unaware of the previous results. The coefficient of variation for positive strain in the four-chamber view was $10.2 \%$ (intraobserver) and $18.8 \%$ (interobserver) and for positive strain rate in four-chamber view was $9.4 \%$ (intraobserver) and $13.7 \%$ (interobserver).

2.5. Statistical Analysis. Continuous variables are expressed as mean \pm standard deviation, while discrete variables are expressed as frequency (percentage). All echocardiographic variables were tested for normality using the Kolmogorov-Smirnov test. Between-group comparisons were conducted using the Student's $t$-test for data with normal distribution and using the Mann-Whitney test for data with nonnormal distribution. Logistic regression models were used to evaluate the association between binary and continuous variables and examine the performance of different predictive models. Receiver operating characteristic (ROC) curves were analyzed to identify the optimal cutoff values of echocardiographic variables for predicting the presence of LAAT, and the results were expressed as the area under the ROC curve (AUC). The quality of the models was expressed in terms of odds ratios (ORs) with 95\% confidence intervals (95\% CIs). Bland-Altman analysis was conducted to assess intraobserver and interobserver variability. $p$ values $\leq 0.05$ were considered to indicate statistical significance.

\section{Results}

3.1. Characteristics of the Study Population. From September 2013 to December 2017, we enrolled 181 patients who had no history of using antithrombotic or antiplatelet therapy. However, 37 (20.4\%) patients were excluded from the study because of unacceptable quality of transthoracic images. All remaining patients $(n=144)$ underwent TEE successfully. Among these, 35 (24.3\%) patients were found to have LAAT.
The demographic, clinical, and left ventricular echocardiographic characteristics of the 144 patients included in the final analysis are summarized in Table 1.

Upon stratifying the patients according to the presence of LAAT (with vs without LAAT), the two groups did not differ in terms of average age, body mass index, or left ventricular dimensions, volume, and ejection fraction. However, the prevalence of hypertension was higher among patients with LAAT than among those without LAAT $(82.9 \%$ vs $64.2 \%)$, as was the prevalence of ischemic stroke (45.7\% vs $23.9 \%$ ). Other risk factors (vascular diseases, heart failure, and diabetes) did not differ between the two groups.

3.2. LA Parameters. We found that LA parameters on echocardiography (diameter, area, volume, and volume index) were larger in nonvalvular AF patients with LAAT than in those without LAAT. On the contrary, LA parameters on STE (positive strain, positive strain rate, and negative strain rate in the two- and four-chamber views) were lower in patients with LAAT than in those without LAAT. All differences were statistically significant (Table 2).

We conducted ROC curve analysis to evaluate the diagnostic value of LA echocardiographic parameters for LAAT prediction in patients with chronic nonvalvular AF. The highest diagnostic performance was noted for LA positive strain in the four-chamber view (optimal cutoff, $\leq 6.7 \%$; $\mathrm{AUC}=0.84$; sensitivity $=77.1 \%$; specificity $=77.1 \%$ ), whereas the lowest performance was noted for LA anteriorposterior diameter (optimal cutoff, $>5.7 \mathrm{~cm}$; $\mathrm{AUC}=0.65$; sensitivity $=94.3 \%$; specificity $=32.1 \%$ ). The results of the ROC curve analysis for all LA parameters are summarized in Table 3. All LA parameters had good AUC, ranging from 0.71 (LA area in the four-chamber view $>26.7 \mathrm{~cm}^{2}$; LA positive strain rate in the two-chamber view $\leq 0.58 \mathrm{~s}^{-1}$; LA negative strain rate in the four-chamber view $>-0.94 \mathrm{~s}^{-1}$ ) to 0.83 (LA negative strain rate in the four-chamber view $\left.>-0.73 \mathrm{~s}^{-1}\right)$.

Using logistic regression analysis and ROC curve analysis, we were able to test several models for LAAT prediction (Figure 2). The model based solely on the 
TAвle 1: Demographic, clinical, and echocardiographic characteristics of patients with nonvalvular atrial fibrillation.

\begin{tabular}{lccc}
\hline Characteristic & With LAAT $(n=35)$ & Without LAAT $(n=109)$ & $p$ value \\
\hline Female sex & $6(11.7 \%)$ & $24(22 \%)$ & $<8.4 \pm 10.6$ \\
Age, years & $72.4 \pm 11.5$ & $22.5 \pm 3.1$ & 0.20 \\
BMI $\left(\mathrm{kg} / \mathrm{m}^{2}\right)$ & $22.1 \pm 2.1$ & $78.4 \pm 11.5$ & 0.01 \\
Heart rate $(\mathrm{bpm})$ & $76.3 \pm 10.5$ & $31(28.4 \%)$ & 0.33 \\
Heart failure & $10(28.6 \%)$ & $70(64.2 \%)$ & $<0.001$ \\
Hypertension & $29(82.9 \%)$ & $17(15.6 \%)$ & $<0.001$ \\
Diabetes type 2 & $4(11.4 \%)$ & $26(23.9 \%)$ & $<0.001$ \\
History of ischemic stroke & $16(45.7 \%)$ & $20(18.3 \%)$ & $<.001$ \\
Vascular disease & $7(20 \%)$ & $49.7 \pm 1.6$ & $<.5$ \\
CHA ${ }_{2}$ DS ${ }_{2}$-VASc score & $3.63 \pm 2.0$ & $35.3 \pm 9.3$ & 0.001 \\
LV diastolic diameter $(\mathrm{mm})$ & $50.4 \pm 7.2$ & $121.3 \pm 51.6$ & 0.08 \\
LV systolic diameter $(\mathrm{mm})$ & $36.8 \pm 8.2$ & $58.2 \pm 4.04$ & 0.31 \\
LV diastolic volume $(\mathrm{mL})$ & $124.7 \pm 43$ & $56 \pm 13.6$ & 0.29 \\
LV systolic volume $(\mathrm{mL})$ & $62 \pm 34.8$ & $47.1 \pm 11.6$ & 0.47 \\
LV ejection fraction $(\%)$ & & 0.36 \\
\hline
\end{tabular}

Data represent frequency (percentage) or mean \pm standard deviation. BMI: body mass index; LAAT: left atrial appendage thrombus; LV: left ventricle.

TABLE 2: Left atrial strain and strain rate parameters in patients with nonvalvular atrial fibrillation.

\begin{tabular}{|c|c|c|c|}
\hline LA parameter on TTE & With LAAT $(n=35)$ & Without LAAT $(n=109)$ & $p$ value \\
\hline Diameter, $\mathrm{cm}$ & $6.49 \pm 0.59$ & $6.13 \pm 0.77$ & 0.015 \\
\hline Area in two-chamber view $\left(\mathrm{cm}^{2}\right)$ & $28.51 \pm 4.17$ & $24.33 \pm 5.54$ & $<0.001$ \\
\hline Area in four-chamber view $\left(\mathrm{cm}^{2}\right)$ & $30.0 \pm 5.32$ & $26.35 \pm 5.43$ & 0.001 \\
\hline Volume (mL) & $111.79 \pm 30.42$ & $88.33 \pm 29.02$ & $<0.001$ \\
\hline Volume index $\left(\mathrm{mL} / \mathrm{m}^{2}\right)$ & $69.59 \pm 21.17$ & $55.78 \pm 20.16$ & 0.001 \\
\hline Positive strain in two-chamber view (\%) & $5.68 \pm 1.91$ & $10.61 \pm 6.92$ & $<0.001$ \\
\hline Positive strain in four-chamber view (\%) & $5.28 \pm 2.54$ & $10.47 \pm 5.31$ & $<0.001$ \\
\hline Positive strain rate in two-chamber view $\left(\mathrm{s}^{-1}\right)$ & $0.52 \pm 0.16$ & $0.7 \pm 0.31$ & $<0.001$ \\
\hline Positive strain rate in four-chamber view $\left(\mathrm{s}^{-1}\right)$ & $0.51 \pm 0.15$ & $0.69 \pm 0.26$ & $<0.001$ \\
\hline Negative strain rate in two-chamber view $\left(\mathrm{s}^{-1}\right)$ & $-0.63 \pm 0.19$ & $-0.91 \pm 0.45$ & $<0.001$ \\
\hline Negative strain rate in four-chamber view $\left(\mathrm{s}^{-1}\right)$ & $-0.57 \pm 0.18$ & $-0.98 \pm 0.41$ & $<0.001$ \\
\hline
\end{tabular}

Data represent mean \pm standard deviation. LA: left atrial; LAAT: left atrial appendage thrombus; TEE: transthoracic echocardiography.

TABle 3: Diagnostic value of LA echocardiographic parameters for LAAT prediction.

\begin{tabular}{|c|c|c|c|c|c|c|}
\hline LA parameter & Cutoff value & Sensitivity (\%) & Specificity (\%) & AUC & $95 \% \mathrm{CI}$ & $p$ value \\
\hline Area in four-chamber view $\left(\mathrm{cm}^{2}\right)$ & $>26.7$ & 74.3 & 63.3 & 0.71 & $0.63-0.78$ & $<0.001$ \\
\hline Area in two-chamber view $\left(\mathrm{cm}^{2}\right)$ & $>26.8$ & 71.4 & 75.2 & 0.76 & $0.68-0.83$ & $<0.001$ \\
\hline Diameter $(\mathrm{cm})$ & $>5.7$ & 94.3 & 32.1 & 0.65 & $0.57-0.73$ & $<0.001$ \\
\hline Volume (mL) & $>99.8$ & 68.6 & 73.4 & 0.73 & $0.65-0.80$ & $<0.001$ \\
\hline Volume index $\left(\mathrm{mL} / \mathrm{m}^{2}\right)$ & $>57.0$ & 77.1 & 64.2 & 0.72 & $0.64-0.79$ & $<0.001$ \\
\hline Positive strain in two-chamber view (\%) & $\leq 7.81$ & 91.4 & 67.0 & 0.81 & $0.74-0.87$ & $<0.001$ \\
\hline Positive strain in four-chamber view (\%) & $\leq 6.7$ & 77.1 & 77.1 & 0.84 & $0.76-0.89$ & $<0.001$ \\
\hline Positive strain rate in two-chamber view $\left(\mathrm{s}^{-1}\right)$ & $\leq 0.58$ & 74.3 & 60.6 & 0.71 & $0.63-0.78$ & $<0.001$ \\
\hline Positive strain rate in four-chamber view $\left(\mathrm{s}^{-1}\right)$ & $\leq 0.55$ & 65.7 & 72.5 & 0.75 & $0.67-0.82$ & $<0.001$ \\
\hline Negative strain rate in two-chamber view $\left(\mathrm{s}^{-1}\right)$ & $>-0.94$ & 100 & 44.0 & 0.71 & $0.63-0.78$ & $<0.001$ \\
\hline Negative strain rate in four-chamber view $\left(\mathrm{s}^{-1}\right)$ & $>-0.73$ & 85.7 & 70.6 & 0.83 & $0.76-0.89$ & $<0.001$ \\
\hline
\end{tabular}

Data were obtained using receiver operating characteristic curve analysis. 95\% CI: 95\% confidence interval; AUC: area under the curve; LA: left atrial; LAAT: left atrial appendage thrombus.

$\mathrm{CHA}_{2} \mathrm{DS}_{2}$-VASc score (model 1) revealed no association between this parameter and LAAT $(\mathrm{OR}=1.24 ; 95 \% \mathrm{CI}$, $0.99-1.55)$, providing low predictive ability $\left(\chi^{2}=3.53\right)$. The model considering the LAVi in addition to the $\mathrm{CHA}_{2} \mathrm{DS}_{2}-$ VASc score (model 2) revealed a significant association between LAAT and the combination of these parameters $(\mathrm{OR}=1.03 ; 95 \% \mathrm{CI}, 1.01-1.05 ; p<0.01)$, providing higher predictive ability $\left(\chi^{2}=12.03\right)$. Adding STE-based LA parameters further increased the quality of the predictive models for LAAT detection. Specifically, models involving the $\mathrm{CHA}_{2} \mathrm{DS}_{2}$-VASc score, LAVi, and LA positive strain in the four-chamber view (model 3) or LA negative strain rate in the two-chamber view (model 4) revealed significant associations between LAAT and these combinations of parameters, providing high but comparable predictive ability ( $\chi^{2}: 33.47$ and 33.48 , respectively) (Table 4$)$. 


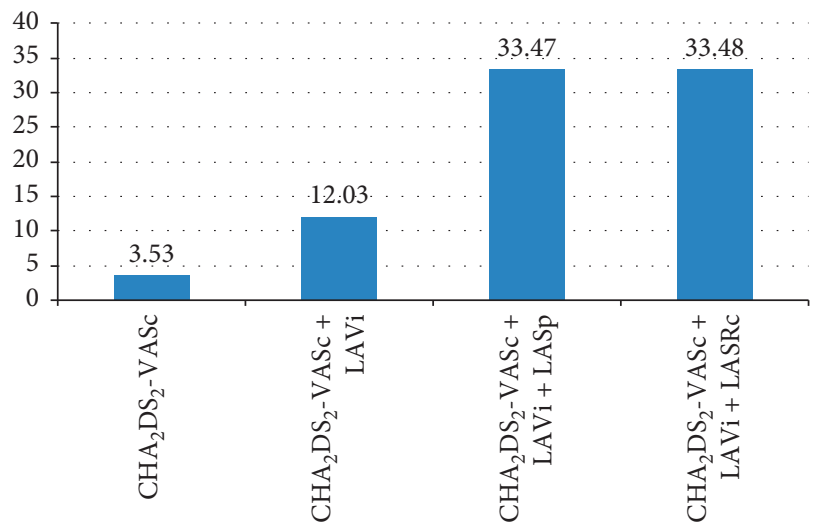

FIgURE 2: Performance of predictive models based on the $\mathrm{CHA}_{2} \mathrm{DS}_{2}$-VASc score and left atrial speckle tracking parameters for LAAT prediction.

TABLE 4: Models of combined CHA2DS2-VASc score and left atrial speckle tracking parameters for LAA thrombus prediction.

\begin{tabular}{|c|c|c|c|c|}
\hline$x^{2}$ & Model $1\left(\chi^{2}=3.53\right)$ & Model $2\left(\chi^{2}=12.03\right)$ & Model $3\left(\chi^{2}=33.47\right)$ & Model $4\left(\chi^{2}=33.48\right)$ \\
\hline \multirow{5}{*}{ Variables } & $\mathrm{CHA}_{2} \mathrm{DS}_{2}-\mathrm{VASc}$ & $\mathrm{CHA}_{2} \mathrm{DS}_{2}-\mathrm{VASc}+\mathrm{LAVi}$ & $\begin{array}{c}\mathrm{CHA}_{2} \mathrm{DS}_{2^{-}} \\
\mathrm{VASc}+\mathrm{LAVi}+\mathrm{LASp}(4 \mathrm{C})\end{array}$ & $\begin{array}{c}\mathrm{CHA}_{2} \mathrm{DS}_{2^{-}} \\
\mathrm{VASc}+\mathrm{LAVi}+\mathrm{LASRc}(2 \mathrm{C})\end{array}$ \\
\hline & $\begin{array}{c}\mathrm{OR}=1.24(95 \% \mathrm{CI}: \\
0.99-1.55)\end{array}$ & $\mathrm{OR}=1.15(95 \% \mathrm{CI}: 0.91-1.46)$ & $\mathrm{OR}=1.10(95 \%$ CI: $0.85-1.43)$ & $\mathrm{OR}=1.10(95 \% \mathrm{CI}: 0.85-1.43)$ \\
\hline & - & 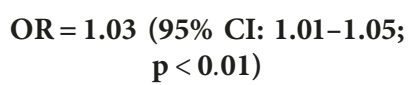 & $\mathrm{OR}=1.02(95 \% \mathrm{CI}: 0.85-1.43)$ & $\mathrm{OR}=1.02(95 \% \mathrm{CI}: 0.85-1.43)$ \\
\hline & - & 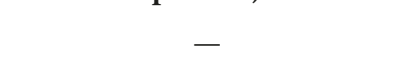 & 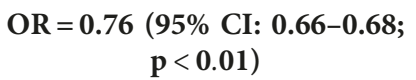 & $\begin{array}{c}\mathrm{OR}=0.76(95 \% \mathrm{CI}: 0.65-0.90 ; \\
\mathrm{p}<0.01)\end{array}$ \\
\hline & - & - & -1 & 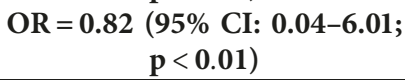 \\
\hline
\end{tabular}

95\% CI: 95\% confidence interval; LAVi: left atrial volume index; LASp(4C): left atrial positive strain in four-chamber view; LASRc(2C): left atrial negative strain rate in two-chamber view; OR: odds ratio.

\section{Discussion}

Many patients referred to our tertiary hospital for chronic AF had no history of antithrombotic or antiplatelet therapy, which often means that the patients were not aware of their disorder or that their general practitioners did not prescribe adequate therapy based on the $\mathrm{CHA}_{2} \mathrm{DS}_{2}$-VASc score $[23,24]$. Moreover, this group of patients often presented with persistent episodes of atrial tachyarrhythmia which were associated with elevated asymmetric dimethylarginine (ADMA) and downregulates endothelial nitric oxide synthase (eNOS). These factors were shown to be the risks of oxidative stress, vascular injury, and endothelial dysfunction [25]. This might be the reason why the prevalence of LAAT was higher in our study $(24.3 \%)$ than in the previous studies $[26,27]$.

Thromboembolism is the most common complication in patients with chronic nonvalvular AF. While TEE is the gold standard tool to detect LAAT [28-31], this modality is semiinvasive and may not be applicable in all cases. Recent studies reported the successful use of noninvasive approaches to diagnose LAAT, including two-dimensional STE-based measurement of LA parameters [32, 33]. However, these models are complex and not easy to apply in clinical practice. Therefore, we set out to develop simple predictive models for LAAT detection.
Recent guidelines recommend using the $\mathrm{CHA}_{2} \mathrm{DS}_{2}$ VASc score to predict stroke risk in AF patients $[15,34]$, as high $\mathrm{CHA}_{2} \mathrm{DS}_{2}$-VASc score $(\geq 4)$ was shown to be associated with thromboembolism in nonvalvular AF patients [35]. However, in our study, the mean $\mathrm{CHA}_{2} \mathrm{DS}_{2}$-VASc score in both groups was below 4 (with LAAT, $3.63 \pm 2.0$; without LAAT, $3.0 \pm 1.6$; Table 1). In other words, the $\mathrm{CHA}_{2} \mathrm{DS}_{2^{-}}$ VASc score alone cannot be used to predict LAAT in treatment-naïve patients with chronic nonvalvular AF (see also Figure 2). Therefore, we tried to increase the predictive value of $\mathrm{CHA}_{2} \mathrm{DS}_{2}$-VASc score-based models by adding LA echocardiographic parameters. Indeed, the combination of $\mathrm{CHA}_{2} \mathrm{DS}_{2}$-VASc score with LA parameters, especially with strain parameters, had a significant association with LAAT and provided high predictive ability (models 3 and 4; Figure 2).

Upon ROC curve analysis, we found good diagnostic performance for LA positive longitudinal strain in the fourchamber view (optimal cutoff, $\leq 6.7 \%$; $\mathrm{AUC}=0.84$; sensitivity $=77.1 \%$; specificity $=77.1 \%$ ), negative longitudinal strain rate in the four-chamber view (optimal cutoff, $\geq 0.73 \mathrm{~s}^{-1} ; \quad$ AUC $=0.83 ; \quad$ sensitivity $=85.7 \%$; $\quad$ specificity $=70.6 \%$ ), and LAVi (optimal cutoff, $\geq 57 \mathrm{~mL} / \mathrm{m}^{2}$; $\mathrm{AUC}=0.72$; sensitivity $=77.1 \%$; specificity $=64.5 \%)$. These results suggest that the diagnostic performance for LAAT detection in patients with chronic nonvalvular AF may be 
higher when using the combination of such LA-based parameters than when using the $\mathrm{CHA}_{2} \mathrm{DS}_{2}$-VASc score alone.

We found that the $\mathrm{CHA}_{2} \mathrm{DS}_{2}$-VASc score was not associated with LAAT (Figure 2), which is in agreement with the findings of Sugiura et al. [36] and Tang et al. [37]. However, by adding LAVi to the $\mathrm{CHA}_{2} \mathrm{DS}_{2}$-VASc score, we could increase the predictive ability of the model $\left(\chi^{2}\right)$ from 3.53 to 12.53 . Further addition of an LA strain parameter provided a substantial increase in predictive power (to 33.47 with the addition of LA positive strain, and to 33.48 with the addition of LA negative strain rate). These results can be explained by the fact that, in chronic nonvalvular AF, LA dilatation and dysfunction is the primary contributor to clot formation [38]. Other authors also proposed models combining clinical and echocardiographic parameters. For example, Obokata et al. proposed models that combined $\mathrm{CHA}_{2} \mathrm{DS}_{2}$-VASc score, oral anticoagulant use, left ventricular ejection fraction, and LA total longitudinal strain to increase predictive value [33]. The advantage of the models proposed in our present study is that they achieve good predictive ability using only echocardiographic parameters, thus being easier to apply in clinical practice.

There are several limitations to our study. Firstly, this was a single-center study enrolling a small number of patients without anticoagulation treatment. Secondly, the software we used to measure LA dimensions and strain (EchoPAC; GE Medical Systems) was specifically designed for evaluating the left ventricle, not the LA. Lastly, STE parameters vary across ultrasound machine manufacturers, which may preclude direct comparison with previously reported values.

\section{Conclusions}

We propose two novel and simple models for noninvasive LAAT prediction in patients with chronic nonvalvular AF. These models combine the $\mathrm{CHA}_{2} \mathrm{DS}_{2}$-VASc score with LAVi and LA longitudinal strain parameters (positive strain or negative strain rate) measured on STE in the four-chamber view. We hope these simple models can help with decisionmaking in managing the antithrombotic treatment of such patients, whose risk of stroke cannot be determined solely based on the $\mathrm{CHA}_{2} \mathrm{DS}_{2}$-VASc score.

\section{Data Availability}

The data used to support the findings of this study are available from the corresponding author upon request.

\section{Disclosure}

All activities were conducted as part of the authors' employment relationship with the Military Heart Institute.

\section{Conflicts of Interest}

The authors declare that there are no conflicts of interest regarding the publication of this paper.

\section{References}

[1] M. Zoni-Berisso, F. Lercari, T. Carazza, and S. Domenicucci, "Epidemiology of atrial fibrillation: European perspective," Clinical Epidemiology, vol. 6, pp. 213-220, 2014.

[2] A. S. Go, E. M. Hylek, K. A. Phillips et al., "Prevalence of diagnosed atrial fibrillation in adults," JAMA, vol. 285 , no. 18 , pp. 2370-2375, 2001.

[3] Y. Iguchi, K. Kimura, J. Aoki et al., "Prevalence of atrial fibrillation in community-dwelling Japanese aged 40 Years or older in Japan," Circulation Journal, vol. 72, no. 6, pp. 909913, 2008.

[4] K. S. Lee, S. J. Choi, S. H. Park et al., "Prevalence of atrial fibrillation in middle-aged people in Korea: the Korean genome and epidemiology study," Korean Circulation Journal, vol. 38, no. 11, pp. 601-605, 2008.

[5] P. A. Wolf, R. D. Abbott, and W. B. Kannel, "Atrial fibrillation as an independent risk factor for stroke: the Framingham study," Stroke, vol. 22, no. 8, pp. 983-988, 1991.

[6] H. S. Markus, U. Khan, J. Birns et al., "Differences in stroke subtypes between black and white patients with stroke," Circulation, vol. 116, no. 19, pp. 2157-2164, 2007.

[7] P. Kirchhof, A. Auricchio, J. Bax et al., "Outcome parameters for trials in atrial fibrillation: executive summary: recommendations from a consensus conference organized by the German Atrial Fibrillation Competence NETwork (AFNET) and the European Heart Rhythm Association (EHRA)," European Heart Journal, vol. 28, no. 22, pp. 2803-2817, 2007.

[8] S. Y. Ho, K. P. McCarthy, and F. F. Faletra, "Anatomy of the left atrium for interventional echocardiography," European Journal of Echocardiography, vol. 12, no. 10, 2011.

[9] A. Faustino, R. Providencia, S. Barra et al., "Which method of left atrium size quantification is the most accurate to recognize thromboembolic risk in patients with non-valvular atrial fibrillation?," Cardiovasc Ultrasound, vol. 12, no. 28, pp. 1476-7120, 2014.

[10] R. Vianna-Pinton, C. A. Moreno, C. M. Baxter, K. S. Lee, T. S. Tsang, and C. P. Appleton, "Two-dimensional speckletracking echocardiography of the left atrium: feasibility and regional contraction and relaxation differences in normal subjects," Journal of the American Society of Echocardiography, vol. 22, no. 3, pp. 229-305, 2009.

[11] A. C. Y. To, S. D. Flamm, T. H. Marwick, and A. L. Klein, "Clinical utility of multimodality LA imaging," JACC: Cardiovascular Imaging, vol. 4, no. 7, pp. 788-798, 2011.

[12] W. P. Abhayaratna, J. B. Seward, C. P. Appleton et al., "Left atrial size," Journal of the American College of Cardiology, vol. 47, no. 12, pp. 2357-2363, 2006.

[13] H. C. Dittrich, L. A. Pearce, R. W. Asinger et al., "Left atrial diameter in nonvalvular atrial fibrillation: an echocardiographic study," American Heart Journal, vol. 137, no. 3, pp. 494-499, 1999.

[14] F. Islas, C. Olmos, C. Vieira et al., "Thromboembolic risk in atrial fibrillation: association between left atrium mechanics and risk scores. A study based on 3D wall-motion tracking technology," Echocardiography, vol. 32, no. 4, pp. 644-653, 2015.

[15] A. J. Camm, P. Kirchhof, G. Y. Lip et al., "Guidelines for the management of atrial fibrillation: the task force for the management of atrial fibrillation of the european society of Cardiology (ESC)," EP Europace, vol. 12, no. 10, pp. 13601420, 2010.

[16] E. Donal, G. Y. H. Lip, M. Galderisi et al., "EACVI/EHRA Expert Consensus Document on the role of multi-modality 
imaging for the evaluation of patients with atrial fibrillation," European Heart Journal-Cardiovascular Imaging, vol. 17, no. 4, pp. 355-383, 2016.

[17] R. M. Lang, L. P. Badano, V. Mor-Avi et al., "Recommendations for cardiac chamber quantification by echocardiography in adults: an update from the American society of echocardiography and the European association of cardiovascular imaging," Journal of the American Society of Echocardiography, vol. 28, no. 1, pp. 1-39, 2015.

[18] V. Mor-Avi, R. M. Lang, L. P. Badano et al., "Current and evolving echocardiographic techniques for the quantitative evaluation of cardiac mechanics: ASE/EAE consensus statement on methodology and indications endorsed by the Japanese society of echocardiography," European Journal of Echocardiography, vol. 12, no. 3, pp. 167-205, 2011.

[19] J.-U. Voigt, G. Pedrizzetti, P. Lysyansky et al., "Definitions for a common standard for $2 \mathrm{D}$ speckle tracking echocardiography: consensus document of the EACVI/ASE/Industry task force to standardize deformation imaging," Journal of the American Society of Echocardiography, vol. 28, no. 2, pp. 183-193, 2015.

[20] H. Motoki, A. Dahiya, M. Bhargava et al., "Assessment of left atrial mechanics in patients with atrial fibrillation: comparison between two-dimensional speckle-based strain and velocity vector imaging," Journal of the American Society of Echocardiography, vol. 25, no. 4, pp. 428-435, 2012.

[21] R. M. Saraiva, S. Demirkol, A. Buakhamsri et al., "Left atrial strain measured by two-dimensional speckle tracking represents a new tool to evaluate left atrial function," Journal of the American Society of Echocardiography, vol. 23, no. 2, pp. 172-180, 2010.

[22] D. Fatkin, R. P. Kelly, and M. P. Feneley, "Relations between left atrial appendage blood flow velocity, spontaneous echocardiographic contrast and thromboembolic risk in vivo," Journal of the American College of Cardiology, vol. 23, no. 4, pp. 961-969, 1994.

[23] V. Fuster, L. E. Ryden, D. S. Cannom et al., “ACCF/AHA/HRS focused updates incorporated into the ACC/AHA/ESC 2006 guidelines for the management of patients with atrial fibrilation: a report of the American College of Cardiology Foundation/American Heart Association Task Force on practice guidelines," Circulation, vol. 123, no. 10, pp. e269e367, 2011.

[24] P. Kirchhof, S. Benussi, D. Kotecha et al., "ESC Guidelines for the management of atrial fibrillation developed in collaboration with EACTS," EP Europace, vol. 27, no. 11, pp. 16091678, 2016.

[25] A. Goette, M. Hammwöhner, A. Bukowska et al., "The impact of rapid atrial pacing on ADMA and endothelial NOS," International Journal of Cardiology, vol. 154, no. 2, pp. 141-146, 2012.

[26] R. Malik, D. M. Alyeshmerni, Z. Wang et al., "Prevalence and predictors of left atrial thrombus in patients with atrial fibrillation: is transesophageal echocardiography necessary before cardioversion?," Cardiovascular Revascularization Medicine, vol. 16, no. 1, pp. 12-14, 2015.

[27] G. Corrado, S. Beretta, L. Sormani et al., "Prevalence of atrial thrombi in patients with atrial fibrillation/flutter and subtherapeutic anticoagulation prior to cardioversion," European Journal of Echocardiography, vol. 5, no. 4, pp. 257-261, 2004.

[28] R. T. Hahn, T. Abraham, M. S. Adams et al., "Guidelines for performing a comprehensive transesophageal echocardiographic examination: recommendations from the American society of echocardiography and the society of cardiovascular anesthesiologists," Journal of the American Society of Echocardiography, vol. 26, no. 9, pp. 921-964, 2013.

[29] Y. Agmon, B. K. Khandheria, F. Gentile, and J. B. Seward, "Echocardiographic assessment of the left atrial appendage," Journal of the American College of Cardiology, vol. 34, no. 7, pp. 1867-1877, 1999.

[30] D. Y. Leung, P. M. Davidson, G. B. Cranney, and W. F. Walsh, "Thromboembolic risks of left atrial thrombus detected by transesophageal echocardiogram," The American Journal of Cardiology, vol. 79, no. 5, pp. 626-629, 1997.

[31] W. J. Manning, R. M. Weintraub, C. A. Waksmonski et al., "Accuracy of transesophageal echocardiography for identifying left atrial thrombi: a prospective, intraoperative study," Annals of Internal Medicine, vol. 123, no. 11, pp. 817-822, 1995.

[32] D. P. Leong, E. Joyce, P. Debonnaire et al., "Left atrial dysfunction in the pathogenesis of cryptogenic stroke: novel insights from speckle-tracking echocardiography," Journal of the American Society of Echocardiography, vol. 30, no. 1, pp. 71-79, 2017.

[33] M. Obokata, K. Negishi, K. Kurosawa et al., "Left atrial strain provides incremental value for embolism risk stratification over CHA2DS2-VASc score and indicates prognostic impact in patients with atrial fibrillation," Journal of the American Society of Echocardiography, vol. 27, no. 7, pp. 710-716, 2014.

[34] C. T. January, L. S. Wann, J. S. Alpert et al., "2014 AHA/ACC/ HRS guideline for the management of patients with atrial fibrillation: executive summary," Circulation, vol. 130, no. 23, pp. 2071-2104, 2014.

[35] E. Zhang, T. Liu, Z. Li, J. Zhao, and G. Li, "High CHA2DS2-VASc score predicts left atrial thrombus or spontaneous echo contrast detected by transesophageal echocardiography," International Journal of Cardiology, vol. 184, pp. 540-542, 2015.

[36] S. Sugiura, E. Fujii, M. Senga, E. Sugiura, M. Nakamura, and M. Ito, "Clinical features of patients with left atrial thrombus undergoing anticoagulant therapy," Journal of Interventional Cardiac Electrophysiology, vol. 34, no. 1, pp. 59-63, 2012.

[37] R.-B. Tang, J.-Z. Dong, X.-L. Yan et al., "Serum uric acid and risk of left atrial thrombus in patients with nonvalvular atrial fibrillation," Canadian Journal of Cardiology, vol. 30, no. 11, pp. 1415-1421, 2014.

[38] G. Y. H. Lip, A. Rumley, F. G. Dunn, and G. D. O. Lowe, "Thrombogenesis in mitral regurgitation and aortic stenosis," Angiology, vol. 47, no. 12, pp. 1117-1125, 1996. 


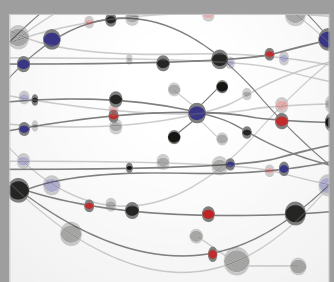

The Scientific World Journal
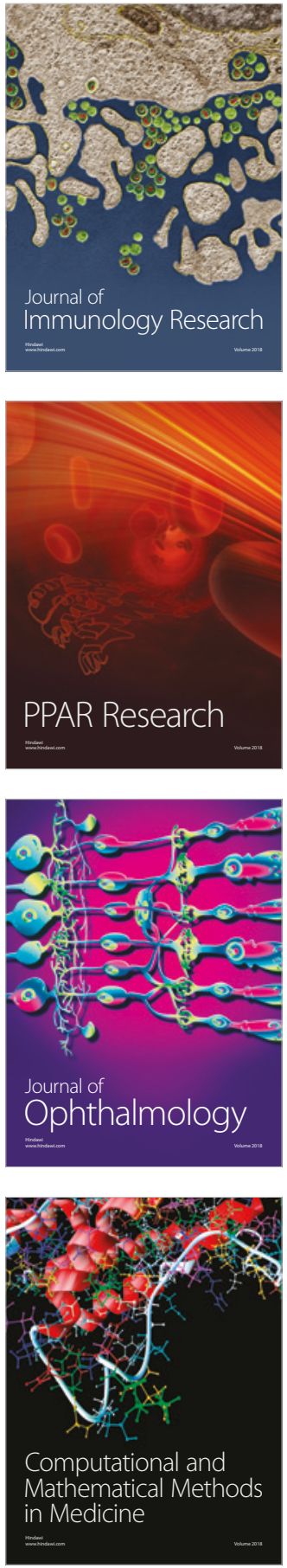

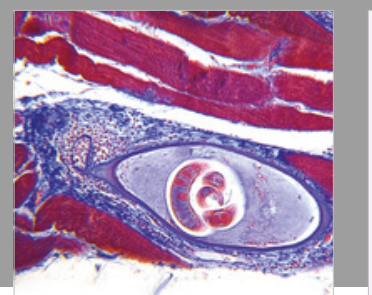

Gastroenterology Research and Practice

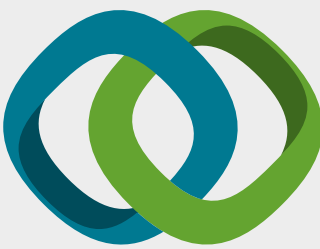

\section{Hindawi}

Submit your manuscripts at

www.hindawi.com
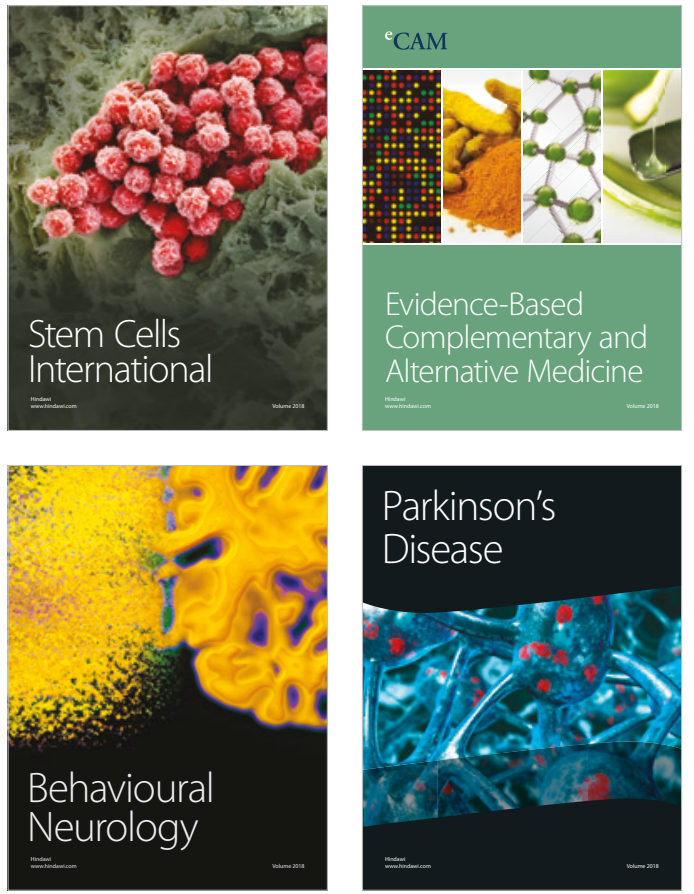

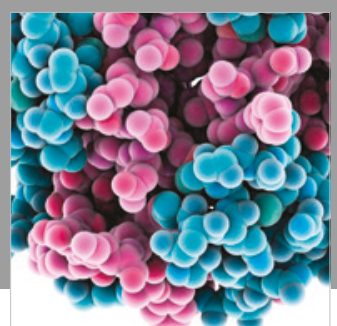

ournal of

Diabetes Research

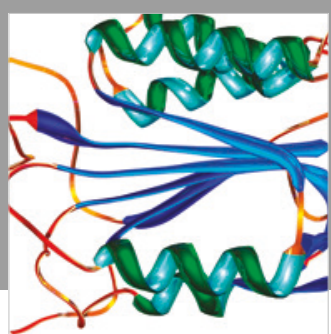

Disease Markers
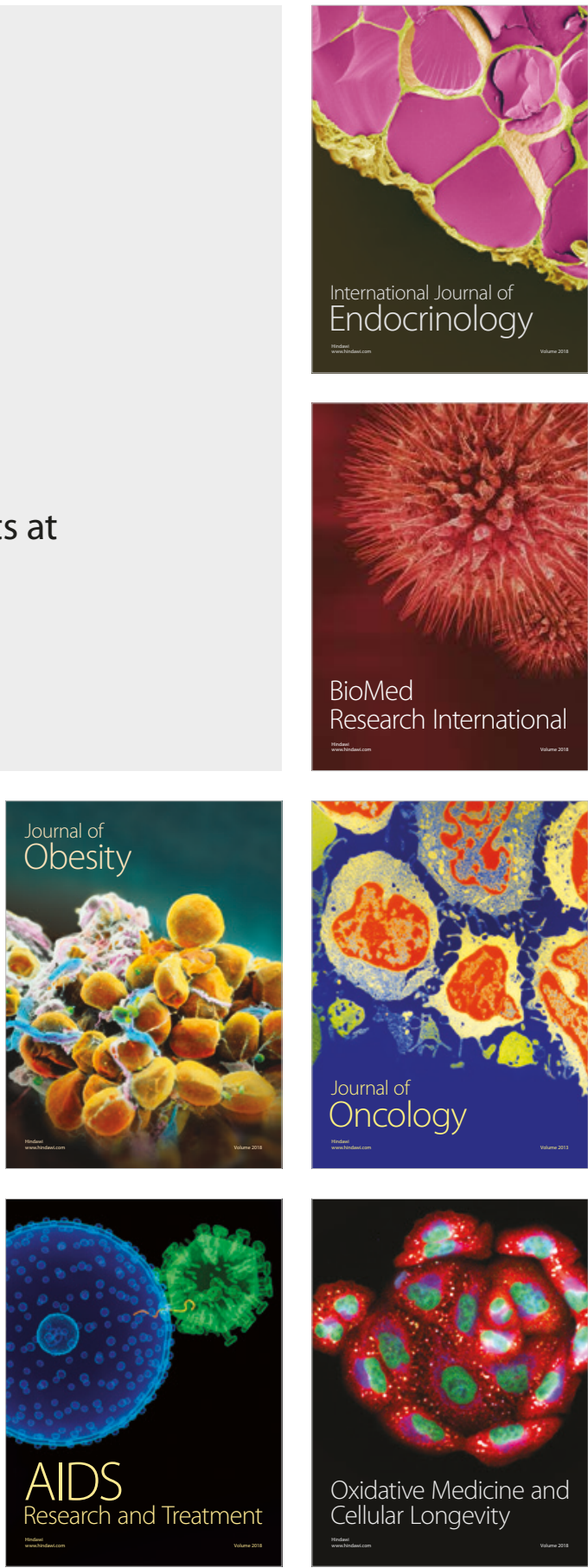